\title{
Die Anstrengungen lohnen sich: \\ Die katholische Presse in der Gegenwart (III*)
}

von K. Rüdiger Durth

„Heute werden im Bereich der Presse, des Hörfunks, des Fernsehens und des Films nicht weniger Anstrengungen und Opfer gefordert, wie sie die mittelalterlichen Städte zur Errichtung ihrer Kathedralen haben leisten müssen." Ein kühner Satz aus der Feder des Präsidenten der „Päpstlichen Kommission für die Instrumente der sozialen Kommunikation", Bischof Andreas Maria Deskur. Geschrieben im Jahr 19771. Im gleichen Jahr befand der sozialdemokratische Bundeskanzler Helmut Schmidt: „Eine spezifische Aufgabe der katholischen Publizistik sehe ich in der Vermittlung zwischen den notwendigerweise divergierenden Auffassungen innerhalb der Gesellschaft."2

Wer wollte bezweifeln, daß die konfessionelle Presse in letzter Zeit an Aufmerksamkeit gewonnen hat? Die Zeiten, in denen sie nur gelegentlich als „Medienmonster im Meinungsmeer der Öffentlichkeit" auftauchte ${ }^{3}$, sind vorbei. Das Interesse beruht auch nicht nur auf der schlagzeilenträchtigen Fusion zwischen „Rheinischer Merkur” und „Deutsche Zeitung”, die im Herbst 1979 angekündigt und zum 1. Januar 1980 vollzogen wurde. Denn ob diese Fusion zu den Anstrengungen zählt, die für die Errichtung von Kathedralen notwendig waren, bleibt dahingestellt. Ein „Publizistischer Dom” nämlich ist diese Wochenzeitung nicht geworden, unter dessen Dach sich alle Katholiken versammeln. Geschweige ein ökumenischer, trotz der Einbringung der einst rein evangelischen „Deutschen Zeitung” für 20 Millionen $\mathrm{DM}^{4}$.

Ein anderer Grund für das Interesse an der konfessionellen und damit auch an der katholischen Presse liegt in der zunehmenden Auseinandersetzung mit den Massenkommunikationsmitteln in ihrer Bedeutung für die Gesellschaft. Die Medienpolitik - neben der Grundwerte-Diskussion wichtigstes innenpolitisches Thema fordert auch die Kirchen heraus. Wollen sie aber in dieser Debatte redlich auftreten, müssen sie zunächst einmal die Gretchenfrage beantworten, wie sie es mit der eigenen Publizistik halten.

Darüber hinaus hat nicht nur die Fach-Öffentlichkeit entdeckt, daß die konfessionelle Publizistik über eine große Auflage verfügt und erheblichen Einfluß ausübt. Je mehr die Kirchen nach ihrem Beitrag in der weithin orientierungslos gewordenen Gesellschaft gefragt werden, desto mehr richtet sich diese Frage auch an die konfessionelle Presse, die sich selbst oft vorwirft, nicht reißerisch genug zu sein. In diesem Zusammenhang ist es bemerkenswert, was Bundeskanzler Helmut Schmidt vor drei Jahren der Katholischen Nachrichten-Agentur (KNA) zum 25jährigen Bestehen schrieb: „Ich frage mich ohnehin, ob es gut ist, wenn unsere Medien überwiegend nur solchen Meldungen Nachrichtenwert beimessen, die von Umstrittenem handeln. Ich frage mich, ob man sich nicht intensiver bemühen sollte, gemeinsam nach Antworten auf die vielfältigen Fragen zu suchen, vor denen unsere Gesellschaft steht; und ob man die gemeinsam gegebenen Antworten nicht mit

\footnotetext{
${ }^{*} \mathrm{~K}$. Rüdiger Durth ist Pastor in der Evangelischen Kirche des Rheinlandes und hauptberufli-
} cher Redakteur der „Bonner Rundschau”. 
gleicher Anstrengung publizieren sollte. Das Zusammenleben der Menschen in unserem Staat kann sich nicht in der gemeinschaftlichen Nutzung des gesellschaftlichen Reichtums erschöpfen: in dieser Beurteilung sind sich der Staat und die Kirchen in diesem Lande einig. Von diesem übereinstimmenden Urteil aus sollte es beiden gelingen, den Dialog darüber, wie der Rahmen der Grundwerte und der Grundrechte - also derjenigen Prinzipien, die das Leben in unserer Gesellschaft menschenwürdig und lebenswert machen - am besten ausgefüllt werden kann, für das Ganze fruchtbar zu machen."6

\section{$K N A$ - von grundlegender Bedeutung}

Katholische Presse der Gegenwart ist ohne die Katholische Nachrichten-Agentur (KNA) nicht mehr denkbar, die Ende 1977 ihr 25jähriges Bestehen feierte und aus diesem Anlaß gewichtige kirchliche und weltliche Glückwünsche erhielt. Da Festreden in unserer Zeit schnell vergessen werden, ist es gut, an einige grundlegende Aussagen zu erinnern. Nicht nur, weil sie die Bedeutung der KNA richtig einschätzen, sondern vor allem deshalb, weil den Worten noch immer die Taten fehlen. Eben auch auf finanziellem Gebiet. Katholische Nachrichtenarbeit kostet nun einmal Geld. Genau das aber wird gern übersehen. Erinnern wir also an einige solcher Glückwunsch-Adressen:

Kardinal-Staatssekretär Jean Villot: „Nicht nur die Katholiken, sondern alle Mitglieder der Gesellschaft haben ein Recht darauf, durch aktuelle und zuverlässige Berichterstattung ein getreues Bild vom vielfältigen Leben der Kirche vermittelt zu bekommen. Um das zu ermöglichen und vor allem eine gute katholische Presse zu gewährleisten, ist eine katholische Nachrichtenagentur von grundlegender Bedeutung."6

Kardinal Joseph Höffner als Vorsitzender der Deutschen Bischofskonferenz: „Die KNA hat in diesen 25 Jahren durch ihre konsequente Arbeit den Ruf einer qualifizierten Agentur begründet, auf die wir heute nicht mehr verzichten können."7

Bischof Andreas Maria Deskur fand, daß die KNA „im Horizont des weltweiten Einsatzes der katholischen Kirche auf dem Gebiet der sozialen Kommunikationsmittel einen bedeutenden Platz" einnehme und daß sie zu einem „noch größeren und wirksameren Einsatz in der Zukunft zu ermutigen" sei. ${ }^{8}$

Weihbischof Wilhelm Wöste: „Es wäre ein Wunder, wenn der KNA keine Fehler unterlaufen wären. Es wäre schier unvorstellbar, daß sie keinen Anstoß erregt hätte. Aber ebenso muß darauf hingewiesen werden, welche unersetzlichen Dienste sie für die Kirche, den deutschen Katholizismus und unser Volk geleistet hat."9

Werfen wir zunächst einen Blick zurück: Die Katholische Nachrichten-Agentur (KNA) konnte auf die Erfahrungen des Kirchlichen Nachrichtendienstes Köln (KND) und den Christlichen Nachrichtendienst München (CND) zurückgreifen, die nach dem Zweiten Weltkrieg entstanden waren. Schon Anfang der 50er Jahre ergab sich die Notwendigkeit, eine bundesweite Agentur aufzubauen, die finanziell und personell den Erfordernissen einer aktuellen katholischen Nachrichtenarbeit gerecht wurde. Am 11. Januar 1953 erschien dann die erste Meldung mit dem Kürzel KNA. Am 12. Januar, also einen Tag später, erklärte die Redaktion unter der Überschrift „Beginn in Gottes Namen” u.a.: „Die KNA nimmt heute in Deutsch- 
land diese Bemühung auf, der andere katholische Agenturen in anderen Ländern dienen. Sie wird dabei allerdings weder ein apologetischer noch ein im eigentlichen Sinne religiöser Dienst sein; sie will der Presse zuverlässig, aktuell und reichhaltig die Nachrichten und Berichte übermitteln, deren sie bedarf. Ihre Berichterstattung wird die Agentur unter die Worte aus einer Presseansprache des gegenwärtigen Papstes Pius XII. stellen: Die Wahrheit ist ohne Leidenschaft und unparteiisch, sie gründet sich auf Tatsachen und nicht auf Phantasie. Die Wahrheit hat keine Angst, bekanntzuwerden, aber sie verlangt danach, nur in dem weißen Licht der Objektivität dargestellt zu werden und nicht in den schillernden Farben des Vorurteils oder der Vermutung."

Schnell eroberte sich die KNA bei Presse und Funk als aktueller Nachrichtenlieferant ein hohes Ansehen. Aber auch innerhalb der katholischen Kirche selbst fand sie viele Abonnenten der unterschiedlichen Briefdienste, und für die Kirchenzeitungen wurde sie zur Hauptagentur. KNA-Archivleiter Dr. Kurt Granel hat ausgerechnet, daß KNA in den Jahren 1967 bis 1977 rund 250.000 Meldungen, Informationen, Berichte und Artikel verbreitet hat - mit insgesamt 45 Millionen Wörtern. $\mathrm{Da} ß \mathrm{KNA}$ enge Verbindungen zu befreundeten Agenturen im Ausland suchte und sucht, versteht sich von selbst.

Bald nach der Gründung wurde die Redaktion von München nach Bonn verlegt, wo sie heute an der Adenauerallee in unmittelbarer Nachbarschaft zum Sekretariat der Deutschen Bischofskonferenz und zum Regierungsviertel ihr Domizil gefunden hat. Dem ersten Chefredakteur Dr. Karl Bringmann (bis 1959) folgte 1961 Dr. Konrad Kraemer.

Das Stammkapital der KNA Gesellschaft mit beschränkter Haftung beträgt 200.000 DM. Gesellschafter sind die Bistümer oder deren Treuhänder zu 50 Prozent und zu je weiteren 25 Prozent die Mitglieder der Arbeitsgemeinschaft Katholische Presse (AKP) und die UNITAS-Verlagsgesellschaft. Der Redaktion ist ein Beirat zugeordnet, der seit 1971 von Weihbischof Wilhelm Wöste (Münster) geleitet wird.

In Frankfurt befindet sich KNA-Pressebild, eine Einrichtung, die nicht nur unersetzlich ist, sondern auch den Anfang 1980 veröffentlichten „Publizistischen Gesamtplan der Evangelischen Kirche in Deutschland"10 anregte, eine ähnliche Einrichtung für den Bereich der evangelischen Publizistik zu fordern. Eines besseren Beweises der Bewährung bedarf es eigentlich nicht, doch sollen die Zahlen ebenfalls genannt sein:

Das Bildarchiv verfügt über rund 250.000 Negative und Fotos, rund 30.000 Farbdias und 5.000 Porträts von Persönlichkeiten des kirchlichen und öffentlichen Lebens. Seit ihrem Bestehen hat KNA-Pressebild über 200.000 Veröffentlichungen in Zeitschriften, Zeitungen und Büchern erzielt' ${ }^{11}$.

Was der frühere Vorsitzende der Arbeitsgemeinschaft Katholische Presse (AKP), Dr. Ferdinand Oertel, 1977 sagte, gilt auch heute noch uneingeschränkt: „In der Zukunft wird KNA eine noch größere Bedeutung für den Kommunikationsfluß innerhalb der Kirche und zwischen Kirche und Welt zukommen als in den vergangenen 25 Jahren. Entscheidend für ihre Effizienz wird weniger die Frage sein, ob ihre Nachrichten 'gefallen' oder 'richtig' im kirchenamtlichen Sinne sind, sondern die Frage, ob sie 'stimmen', das heißt alle Ereignisse, Äußerungen, Meinungen und 
Probleme der Kirche unverkürzt darstellen. Gerade auch in dieser Richtung ist KNA ein weiterer 'Ausbau' zu wünschen."12

\section{Das Bildschirmzeitalter}

Das Bildschirmzeitalter, das in der Presse Bleisatz, Schreibmaschine und Telex verdrängt, wird auch in der KNA-Zentrale (und damit in den Landesredaktionen) seinen Einzug halten. Ohne dieses Mithalten auf technischem Sektor würde die Katholische Nachrichten-Agentur nicht mehr konkurrenzfähig sein. Während der Evangelische Pressedienst (epd) bereits mit der Umstellung auf die elektronische Nachrichtenbeschaffung und -verteilung begonnen hat (Kosten für die Frankfurter Zentrale rund 500.000 DM und für jede Landesredaktion rund 50.000 DM), ist bei KNA eine entsprechende Entscheidung (März 1980) noch nicht gefallen. Aber im Grundsatz richtet man sich bereits darauf ein. Chefredakteur Konrad Kraemer: „Ich denke aber nicht, daß wir eine halbe Million DM ausgeben müssen."13

Der KNA bleibt auch aus einem anderen Grund eine solche Investition nicht erspart: Sie nutzt ebenso wie epd für den aktuellen Nachrichtendienst für die Presse die Sendefrequenzen des Düsseldorfer Sport- und Informationsdienstes (sid), der ebenfalls bald die elektronischen Nachrichtensysteme einführen wird. Dann muß KNA mitziehen, die 1979 5,3 Millionen Wörter verbreitete (1978 waren es 5,1 Millionen) und über einen Jahresetat von rund fünf Millionen DM verfügt. Doch die technischen Investitionen stellen für Dr. Konrad Kraemer nicht das eigentliche Problem dar: „Wir müssen in Menschen investieren.” Das heißt konkret, KNA benötigt mehr Planstellen für Redakteure. Hier aber zeigen sich die Geldgeber mehr als zugeknöpft. Vom Ausbau also noch keine Rede, obwohl er beim silbernen Jubiläum lautstark beschworen und Anfang der 70er Jahre übrigens auch schon zugesagt worden war.

Gegenwärtig verfügt KNA über 24 Redakteurs-Planstellen, einschließlich der römischen Korrespondenten und der Landesredaktionen, die sich in Hamburg, Berlin, Wiesbaden, Freiburg, Stuttgart und München befinden. Der „Westdeutsche Dienst”, praktisch der nordrhein-westfälische Landesdienst, befindet sich ebenfalls in der Bonner Zentrale. Die wichtigsten Dienste von KNA sind: Aktueller Dienst (Inland, Ausland, Vatikan, Kultur), Ökumenische Information, Info-Dienst, Katholische Korrespondenz und Weltkirche aktuell.

Der Anfang der 70er Jahre durch Gutachten untermauerte beschlossene Ausbauplan aber läßt in der Ausführung auf sich warten. Dieser Plan sieht drei große Phasen vor, die nach Wichtigkeit eingestuft worden sind. Im Mittelpunkt steht der personelle Ausbau, um vor allem die qualitativen Leistungen zu verstärken. Aber auch quantitativ müßte KNA mehr leisten, wenn man etwa an die römische Redaktion denkt. Schließlich ist Papst Johannes Paul II. auch journalistisch sehr anstrengend.

Die wichtigsten Ausbaupläne betreffen folgende Gebiete: Größeres Angebot für die Kirchenpresse, Verlegung des „Westdeutschen Dienstes” in die Landeshauptstadt Düsseldorf, Einstellung von „Feuerwehr-Redakteuren” für aktuelle Reportagen, vor allem aber für erkrankte oder sich in Urlaub befindliche Redakteure. Das gilt vor allem für die Landesredaktionen, die sehr knapp besetzt sind. Aber auch eine finanzielle Verstärkung könnte KNA gut gebrauchen: Ganz abgesehen von den Honoraren für ständige und freie Mitarbeiter müßte die Papierqualität der Brief- 
dienste verbessert werden. Würde man eine zweizeilige Schreibweise einführen, die von den meisten Redaktionen wegen der leichteren Korrektur erwünscht wird, müßte man mehr Geld für Papier, Porto haben.

Gleichzeitig wünscht sich Chefredakteur Kraemer einen intensiveren Ausbau der Beziehungen zum Ausland, speziell einen schnelleren Nachrichtenaustausch mit anderen katholischen Agenturen. Daß die Ausbildung qualifizierter ausländischer katholischer Nachrichtenredakteure ebenfalls eine Aufgabe von KNA ist, dürfte unbestritten sein. Klar aber ist auch, daß eine solche Ausbildung viel Geld kostet, für die Chefreakteur Kraemer gegenwärtig aber keine Mittel zur Verfügung stehen.

\section{Die Zwillingskanone}

„Zwillingskanone auf dem christlichen Zeitungsmarkt” überschrieb die „Kölnische Rundschau” ihren Bericht zur Fusion von „Rheinischer Merkur” und „Deutsche Zeitung”, die im Spätherbst 1979 im deutschen Blätterwald für eine medienpolitische Bombe sorgte. Neutral meldete die FAZ „Eine christliche Zeitung weniger”. „Die Zeit” befand: „Keine Elefantenhochzeit”. Der „Kölner Stadtanzeiger” griff zum Bild der Ehe: „Ob diese Ehe klappt, liegt beim Leser”, und der Herausgeber des „Publik-Forum”, Heinz Wilhelm Brockmann, kommentierte unter dem Datum des Allerseelentages 1979: „Die rheinische Meinungsbastion”.

Die wenigen Schlagzeilen sollen genügen, um zu zeigen, daß der neuen christlichen Wochenzeitung „Rheinischer Merkur - Christ und Welt" zumindest dies gelungen war: Aufmerksamkeit und Sensations-Charakter. Inzwischen ist die Zeitung auf dem Markt, aber schon wieder ohne Chefredakteur. Denn Ludolf Hermann (43jähriger Katholik und Leiter der „Deutschen Zeitung”) gab nur zögernd seine Zustimmung zur Übernahme der neuen Redaktion, sagte $a b$, nahm doch an und ließ bereits wenige Wochen nach Erscheinen der „Zwillingskanone” verbreiten, er gehe zu "Capital” als Chefredakteur.

Seit dem Tod von „Publik” im Jahr 1971 hat keine katholische Zeitung soviel Aufsehen erregt wie der ,neue" Merkur. A ber auch in den Jahren zuvor brauchte sich der „Rheinische Merkur" nicht über mangelnde Aufmerksamkeit zu beklagen. 1975 verkaufte der Hamburger „Jahreszeiten-Verlag" 80 Prozent seiner Anteile an dieser Zeitung - die 1814 Joseph von Görres gegründet hatte, Napoleon die „fünfte Großmacht Europas" nannte, und 1946 als katholische Wochenzeitung wieder erschien an die katholische Kirche. Genauer gesagt an die nordrhein-westfälischen Bistümer und die Diözese Freiburg. Für sechs Millionen DM. Daß die treibende Kraft Kölns Erzbischof, Joseph Kardinal Höffner, war, ist kein Geheimnis.

Der „Rheinische Merkur” sollte fortan das Sprachrohr der katholischen Kirche werden. Was „Publik” versagt blieb, wurde auch dem „Rheinischen Merkur” - mit Ausnahme des Geldes - versagt, nämlich eine tragfähige Auflage. Der Traum von der 100.000-Grenze für das knapp 60.000 Exemplare zählende Wochenblatt ging nicht in Erfüllung. Immer wieder mußte die katholische Kirche neue Gelder zuschießen, startete mit Hilfe der Mediendienstleistungsgesellschaft großangelegte Werbekampagnen. Vergebens. Als die Millionen nicht zogen, mußte sich der „Rheinische Merkur" öffnen. Herausgeber Otto B. Roegele erhielt zwei Kollegen: den Präsidenten des Zentralkomitees der deutschen Katholiken, Münchens Kultusminister Hans Maier, und die evangelische Publizistin Christa Mewes. Letztere sollte auf- 
grund ihres (freilich vor allem in katholischen Kreisen) großen Bekanntheitsgrades evangelische Leser anziehen, die man vornehmlich aus dem Stamm der „Deutschen Zeitung" holen wollte. Auch wurde eine größere inhaltliche Öffnung angestrebt. Sowohl politisch als auch konfessionell. Doch Wunsch und Wirklichkeit waren nicht in Übereinstimmung zu bringen. Die für 1980 angestrebte Auflage von 100.000 Exemplaren blieb aus.

$\mathrm{Daß}$ sie dann doch noch erreicht wurde, hätte sich niemand träumen lassen. Freilich auch nicht den eingeschlagenen Weg durch eine Fusion mit eben der „Deutschen Zeitung”. Die nach dem Zweiten Weltkrieg entstandene Wochenzeitschrift „Christ und Welt" mit dem früheren Bundestagspräsidenten Eugen Gerstenmaier als Teilhaber galt als protestantische Zeitung. Es gab eine ungeschriebene Aufteilung: Südlich der Mainlinie las man „Christ und Welt”, nördlich von ihr das damalige "Sonntagsblatt", herausgegeben von Landesbischof Hanns Lilje.

„Publik” öffnete auch der evangelischen Kirche die publizistischen Augen. Die stark defizitären Wochenzeitungen sollten zusammengefaßt werden. Doch die von „Christ und Welt” umgetaufte „Deutsche Zeitung” wollte nicht. Das sich fortan „Deutsches Allgemeines Sonntagsblatt” nennende Lilje-Organ wurde nun offizielle Wochenzeitung der Evangelischen Kirche in Deutschland mit jährlich vier Millionen DM Subvention.

Die „Deutsche Zeitung” zog als erstes bedeutendes deutsches Presseorgan in die Bundeshauptstadt um und fand an der Diplomatenrennbahn ein neues Domizil, das man sich mit „Telefonbau und Normalzeit" teilte. Aber auch im Schatten der großen Politik schlug dem konservativen Protestantenblatt keine günstigere Stunde. Immerhin pendelte man sich bei einer Auflage um die 130.000 Exemplare ein. Als „Die Welt” von Hamburg nach Bonn zog und sich als Nachbar der DZ niederließ, kursierte das Gerücht, „Deutsche Zeitung” und „Welt am Sonntag” wollten fusionieren, Springer plane eine eigene Wochenzeitung als Konkurrenz zu „Die Zeit”. Daraus wurde nichts.

Zwei Millionen DM Defizit soll die „Deutsche Zeitung” ihrem Verleger Georg von Holtzbrinck jährlich eingebracht haben. Der Verleger sah sich um, klopfte angeblich auch beim „Deutschen Allgemeinen Sonntagsblatt” in Hamburg an. In der Not (war es wirklich eine?) verkaufte er - für angeblich 20 Millionen DM an den „Rheinischen Merkur”, in Köln redigiert und in Koblenz verlegt. „Merkur”-Chefredakteur Herwig Gückelhorn (49) mußte gehen, zumal sein DZ-Kollege Hermann als erfolgreicher galt. Außerdem war er katholisch, was sich selbstverständlich günstig fügte.

130.000 „Deutsche Zeitung” und 60.000 „Rheinischer Merkur”gleich 190.000? Diese Rechnung war falsch, nicht nur wegen der längst vorhandenen Doppelbezieher. Die skeptischen Stimmen überwogen denn auch nach dem „Fusions-Schock”, ganz abgesehen von dem Bedauern darüber, daß die deutsche Zeitungslandschaft schon wieder um ein profiliertes Blatt ärmer geworden war. Lesen protestantische Konservative automatisch eine konservative katholische Zeitung? Sicher nicht.

Über die tatsächliche Auflagenentwicklung schweigt man sich aus. Noch mehr über die Personalpolitik. Die Auflage betrug im März 1980 rund 130.000 Exemplare14. Wieviel tatsächlich verkaufte Exemplare sich darunter befinden, ist nicht nachzuweisen. Verständlich, daß man sich zur Zeit noch in Schweigen hüllt, zumal 
man sich auf der Suche nach einem neuen Chefredakteur befindet. Zusätzlichen qualifizierten Redakteuren übrigens auch, da sich die meisten übernommenen Redakteure von der „Deutschen Zeitung” ebenfalls einen anderen Schreibtisch gesucht haben.

Immerhin ist an der 130.000 Auflage das Evangelische Kirchenamt der Bundeswehr nicht ganz unbeteiligt. Es kauft für Soldaten, Offiziere und Dienststellen aus seinem eigenen Etat 5.000 Exemplare. Ganz wohl ist den protestantischen Militärseelsorgern dabei offensichtlich nicht: „Das muß eine Indiskretion aus dem eigenen Hause sein." War es nicht. Der zuständige Öffentlichkeitsreferent im Evangelischen Kirchenamt der Bundeswehr, Dekan Ottemeyer, bestätigt zwar die Nachbarschaftshilfe („Rheinischer Merkur" und Kirchenamt liegen ebenfalls eng beieinander). Diese sei jedoch zunächst auf ein halbes Jahr begrenzt. Dann werde man die Sache erneut überprüfen ${ }^{15}$. Immerhin bezog das Evangelische Kirchenamt der Bundeswehr früher fast 10.000 Exemplare „Deutsche Zeitung” und das Hamburger „Deutsche Allgemeine Sonntagsblatt” hat keinen Grund zur Klage: es liefert auch weiterhin den Militärseelsorgern 13.000 Exemplare, also rund zehn Prozent der Gesamtauflage. Ein interessantes Beispiel für indirekte Pressesubvention, sicherlich nicht das schlechteste.

Verständlich, daß die bitterste Kritik von „Publik-Forum” kommt: „Enorme Geldsummen hat das bischöfliche Unternehmen bislang verschlungen - mehr als man je für die fallengelassene Wochenzeitung „Publik” aufzuwenden bereit war, das man ja angeblich einstellte, weil kein Geld mehr da war... Man baut noch rechtzeitig vor einer sicher sehr polarisierenden Bundestagswahl eine Meinungsbastion auf, die zu den zahlenmäßig großen Wochenzeitungen der Bundesrepublik gehören wird. Sie soll die amtliche Kirchenmeinung verbreiten helfen ... Für die katholische Kirche ist die Suche nach dem freien Spiel journalistischer Arbeit, nach Diskussion und nach alternativen Blättern auf der Ebene der Wochenzeitungen nun wohl für immer beendet."16

\section{Gedämpfte Zuversicht}

Gedämpfte Zuversicht. So läßt sich die Stimmung innerhalb der katholischen Presse der Gegenwart umschreiben ${ }^{17}$. Daß damit nicht alle Auflagensorgen verschwunden sind, versteht sich von selbst. Immerhin betrug der Auflagenschwund der Bistumsblätter im vergangenen Jahr nur noch 0,5 Prozent gegenüber 1978. Dieses gute Ergebnis konnte dadurch erzielt werden, $\mathrm{da} ß$ einige Blätter erstmals einen kräftigen Aufwind erlebten, die Einbußen von bis zu 4,5 Prozent (Kirchenzeitung Aachen) relativierten. Hier ein Überblick über die neuesten Zahlen der 22 Bistumsblätter ${ }^{18}$ :

Bistum

Aachen

Augsburg

Bamberg

Berlin

Eichstätt

Essen
4. Quartal 1978

4. Quartal 1979

$\begin{aligned} 133387 & -4,5 \% \\ 79986 & -1,9 \\ 56645 & -0,5 \\ 14907 & +2,3 \\ 41496 & +1,2 \\ 79063 & +2,1\end{aligned}$




\begin{tabular}{lrrc}
\hline Freiburg & 137445 & 138697 & $+0,9$ \\
Fulda & 18122 & 17677 & $-2,5$ \\
Hildesheim & 36151 & 35286 & $-2,4$ \\
Köln & 150594 & 146069 & $-2,6$ \\
Limburg & 9289 & 29763 & $+1,6$ \\
Mainz & 44935 & 45354 & $+0,9$ \\
München & 95073 & 95336 & $+0,3$ \\
Münster & 210986 & 212882 & $+0,9$ \\
Osnabrück & 59069 & 59706 & $+1,1$ \\
Paderborn & 130628 & 130064 & $-0,4$ \\
Passau & 44953 & 44759 & $-0,4$ \\
Regensburg & 89129 & 89779 & $+0,7$ \\
Rottenburg & 119913 & 118265 & $-1,2$ \\
Speyer & 69783 & 68962 & $-1,2$ \\
Trier & 104958 & 105057 & $+0,1$ \\
Würzburg & 79430 & 78734 & $-0,9$ \\
\hline
\end{tabular}

Der Verlust von knapp 10.000 Exemplaren innerhalb eines Jahres (von 1978 auf 1979) ist zwar nicht besorgniserregend, doch zeigt er an, daß es noch keinen Umschwung gibt. Obwohl man innerhalb der Bistumspresse die Bescheidenheit gelernt hat, darf man sich nicht damit zufriedengeben, daß der Negativtrend sichtlich abgeflaut ist. Auch die Verlust-Prozente hinter dem Komma können sich auf die Jahre verteilt ganz schön summieren. Deshalb dürfte es eine Selbstverständlichkeit sein, in den Bemühungen um mehr Auflage nicht nachzulassen.

Interessant ist auch ein Blick auf die andere katholische Zeitschriftenpresse für den Vergleichszeitraum 4. Quartal 1978 und 4. Quartal 1979. So mußten die fünf Blätter der sogenannten katholischen Sonntagspresse einen Auflagenverlust von 2,6 Prozent hinnehmen - gegenüber 0,3 zum Vorjahr 1977/78. In konkreten Zahlen ausgedrückt: Die Auflage sank von 577.784 auf 562.855 Exemplare. Am stärksten betroffen war die „Christliche Familie”, die fast 9.500 Exemplare (-8,4 \%) einbüßte. Auch die „neue bildpost” mußte im Vergleichszeitraum von ihren 226.607 Exemplaren über 4.000 einbüßen, was einem Prozentsatz von $1,8 \%$ entspricht.

In der Magazinpresse („Leben und Erziehen” sowie „Weltbild”) war ein Auflagenschwund von 5,7\% festzustellen. Dabei hat „Weltbild”, Mitte der 70er Jahre ebenfalls von katholischen Bischöfen stark finanziell gefördert, erneut 8,1 Prozent eingebüßt, was knapp 36.000 Exemplaren entspricht, so daß die Gesamtauflage am Ende 1979 bei etwas über 400.000 Exemplaren lag. Damit hat diese Zeitschrift in den letzten Jahren mehrere hunderttausend Leser verloren.

Interessant freilich, daß die „Deutsche Tagespost” im Vergleichzeitraum ihre Auflage um 3.281 Exemplare auf 37.104 steigern konnte, was einem Prozentsatz von 9,7 enspricht. Kräftig aufgeholt hat die „Junge Zeit” mit 35.314 Exemplaren plus, was einem Prozentsatz von 79 entspricht. Damit ist diese Zeitschrift der absolute 
Spitzenreiter im Vorjahr gewesen, der von knapp 45.000 auf über 80.000 Exemplare anstieg. Aber auch „Frau und Mutter” konnte sich um 14.119 Exemplare auf 602.628 verbessern, was 2,4 Prozent entspricht.

„Auf Wunder läßt sich die Zukunft nicht aufbauen”, erklärte der frühere Vorsitzende der Arbeitsgemeinschaft Katholischer Presse (AKP), Ferdinand Oertel, auf der Mitgliederversammlung dieser Arbeitsgemeinschaft $1978^{19}$. Auch daran hat sich nichts geändert, denn „die größeren Erwartungen, die von immer kritischer werdenden Christen an die Kirchenzeitungen gestellt werden, verlangen einen gezielten redaktionellen Aufbau”20. So bleibt also die Forderung nach einer „auftragsund lesergerechten Weiterentwicklung der Kirchenpresse"2l auf der Tagesordnung.

Dieses Ziel aber ist nicht nur mit gutem Willen der viel zu wenigen kirchlichen Redakteure zu lösen. Das Image der katholischen Presse ist nach wie vor in der breiten Öffentlichkeit nicht gut, auch wenn Heinrich Böll beispielsweise stark übertreibt: „Schauen Sie sich die katholischen Publikationsinstrumente an - immer und immer wieder Ausnahmen, auch in der kirchlichen Presse, vorausgesetzt -, sie sind die bösartigsten Presseerzeugnisse, die Sie sich denken können. Dagegen ist ein Teil der Springer-Presse in seinem krankhaften Schwachsinn fast harmlos. Die sozusagen über allem schwebende theologisch-wissenschaftliche Entwicklung der katholischen Theologie hat mit dieser Gestalt des kirchlichen Lebens nichts zu tun. Sie schwebt darüber, hat nicht den geringsten Einfluß darauf, ist oft genug deren Opfer ....22

Diese Kritik ist überspitzt, trifft deshalb viele nicht. Aber man muß sich mit ihr auseinandersetzen, mehr noch aber mit der Gleichgültigkeit, die der katholischen Presse entgegengebracht wird. Denn es trifft zu, was der Leiter der Werbefachlichen Akademie München, Helmut Rüdinger, im April 1979 auf einer Werbeleitertagung katholischer Zeitschriften und Verlage feststellte, nämlich, daß das Image der religiösen Presse weithin nicht der Wirklichkeit entspricht. Fast alle, die sie negativ beurteilten, hätten sie nicht oder in den letzten zehn bis fünfzehn Jahren nicht mehr gelesen ${ }^{23}$.

\section{Werbung ist nicht anstößig.}

Weithin tut sich die Werbung für die katholische Publizistik schwer. Daß sie intensiviert werden muß und auch neue Wege zu gehen hat, versteht sich von selbst. Auch hier gilt die Mahnung Ferdinand Oertels: ,Ist die Ansprache der Pfarrer und Pfarrgemeinderäte um Mitwirkung bei der Werbung wirklich die Alternative? Jedenfalls nützt eine inhaltliche Öffnung der Kirchenpresse nichts, wenn sie nicht in der Werbung zum Anlaß und Grund für den Bezug herausgestellt wird. Auch etwa die Gründung einer gemeinsamen Werbegesellschaft (die der Aachener EinhardVerlag in Angriff genommen hat, Anm. des Vf.) hat nur dann Sinn, wenn neue Wege der sachgerechten Abonnementsprache gefunden werden. Methodische und finanzielle Mittel genügen auch hier nicht."24

Zum Freiburger Katholikentag 1978 startete die Arbeitsgemeinschaft Katholischer Pressse (AKP) in Zusammenarbeit mit der Mediendienstleistungsgesellchaft (MDG) erstmals eine große Informationskampagne, die nach AKP-Geschäftsführer Bruno Geuter in erster Linie darauf angelegt war, die katholische Presse bekannt zu machen ${ }^{25}$. Gedruckt wurden 203.000 Faltprospekte „Katholische Presse”. 
Davon wurden bis Dezember 1978180.000 Stück verteilt, inzwischen ist die Auflage ganz vergriffen. Wie wurden diese Faltprospekte verteilt? 156.000 an Pfarrämter, 12.000 auf dem Informationstand Katholikentag, 5.500 an katholische Akademien und andere Bildungseinrichtungen, 4.500 an Mitgliedsverlage und der Rest an andere Zielgruppen. Daraufhin wurden bei der AKP-Geschäftsstelle bis Dezember 1978 rund 18.000 Probeexemplare der unterschiedlichsten katholischen Zeitschriften angefordert. Da auch 1979 noch Anfragen eingingen, war also das Interesse als hoch zu bezeichnen. Doch wie wirkte sich dies auf die Abonnentenwerbung aus? Bis März 1979 wahrscheinlich insgesamt 800 Abonnements.

Die Kosten der Aktion beliefen sich auf immerhin 140.000 DM. Davon entfielen auf den Infostand des Freiburger Katholikentages 26.000 DM, eine Anzeige in der Kirchentagsillustrierten auf 12.000 DM, auf den Druck der Faltprospekte 50.000 DM, auf den Druck des Plakates 10.000 DM, auf den Versand 32.000 DM und weitere Drucksachen $9.000 \mathrm{DM}^{26}$. Daraus wird ersichtlich, welche Anstrengungen erforderlich sind, um höchstens 800 Abonnenten zu gewinnen. Dabei darf der Langzeiteffekt einer solchen Informationskampagne nicht unterschätzt werden.

Eine ähnliche Aktion plant die AKP auch zum Katholikentag 1980 in Berlin. Im Rahmen dieses Laientreffens sollen 300.000 Informationsbroschüren verteilt werden. Die Kosten belaufen sich diesmal nach Angaben der AKP auf rund 200.000 DM.

In jeder Pfarrei gibt es sogenannte Schriftenstände. Was lag also näher, diese einmal auf ihre tatsächliche Wirksamkeit für den Verkauf von katholischen Zeitschriften hin zu untersuchen? Dies geschah $1978^{27}$. Die Ergebnisse waren nicht gerade ermutigend, obwohl sich 77 der 100 in der AKP beschlossenen Verlage an der Umfrage beteiligten.

Etwa 45 Prozent der katholischen Zeitschriften haben den Schriftenstand in ihren Vertrieb mit eingeschaltet. Doch nicht einmal fünf Prozent der Verkaufsauflage werden auf diesem Weg umgesetzt. Einige Zeitschriften sind freilich mit bis zu 20 Prozent beteiligt. Die Verkaufsziffern sind sehr unterschiedlich pro Zeitschrift und Zeitschriftenstand. Zwischen einem bis 70 Exemplaren.

In der zusammenfassenden Beurteilung der Umfrageergebnisse durch die AKP. Geschäftsstelle wird festgestellt, daß der Schriftenstand-Verkauf in der katholischen kirchlichen Presse bisher eine geringe Rolle spielt. Für die Bistumszeitungen ist er offensichtlich nur in den Diasporagemeinden von Bedeutung. Sonst wird er eher abgelehnt, da er auf Kosten des Abonnement-Bezuges geht und die Werbung erschweren kann. Einige Organe der Sonntags- und Magazinpresse (vor allem „neue bildpost”) erzielen hingegen über den Schriftenstand beachtliche Verkaufsergebnisse, die oft freilich mit erhöhten Kosten verbunden sind.

Die meisten Beantworter der Umfrage haben dem Schriftenstand trotzdem Bedeutung zugesprochen. Diese wird nicht allein im Verkauf gesehen, sondern auch in den Funktionen Repräsentation, Imagepflege, Orientierung für den Kirchenbesucher, Ansprache von Touristen. Fast einstimmig vertreten die Antworten der Um, frage die Auffassung, daß sich die Effizienz der Schriftenstände durch entsprechende Maßnahmen wesentlich verbessern läßt. An erster Stelle steht dabei die Forderung nach besserer personeller Betreuung. Durch sie verspricht man sich vor allem eine Änderung des weithin unerfreulichen Zustandes der Schriftenstände in den Kirchen. 
Wesentlich für die Werbung neuer Abonnenten und die Erhaltung der Treue bisheriger Leser ist die Verstärkung der sogenannten Leser-Blatt-Bindung. Hinzu kommt die Einrichtung neuer zielgruppen-orientierter Seiten, etwa der „Kinderund Jugendseite" in den Bistumsblättern. Vorbildlich ist die seit einigen Monaten im „Ruhr-Wort” (Essen) erscheinende Kinderseite, die von Pater Hartmut Kriege redigiert wird.

Auch die Beratungsseiten nehmen zunehmend breiteren Raum in der katholischen Presse ein. Hier dient der entsprechende Erfolg der säkularen Publikumszeitschriften als Vorbild, ohne jedoch deren zum Teil bar jeder Ethik, dafür auf Sensation getrimmten Inhalt zu übernehmen. Die christliche Beratung dürfte für die katholische Presse von großem Nachfrage-Interesse sein. Daß sich „Leser-Reisen” ebenfalls positiv auf das Image der katholischen Presse auswirken und die Leser-BlattBindung stärken, dürfte feststehen.

Daß die Leser von Bistumsblättern und anderen Zeitschriften bereit sind, tief in die Tasche zu greifen, um mit „ihrer" Zeitung Not zu lindern, zeigen einige Zahlen aus dem Jahr 1978: Die „neue bildpost”, die über ein eigenes Hilfswerk verfügt, konnte so zwei Millionen DM einsetzen. Vom Urwaldhospital in Kenia angefangen bis hin zur Flüchtlingshilfe in Thailand. Die Leser der Münchener Katholischen Kirchenzeitung brachten 256.000 DM für Missionare, über 100.000 DM für Behinderte, 100.000 DM für Einsame und Arme sowie 60.000 DM für die Erhaltung einer Südtiroler Kirche auf. Der „Pilger” aus Speyer konnte 360.000 DM seiner Leser für die Entwicklungshilfe der katholischen Kirche zur Verfügung stellen und die Leser des Katholischen Sonntagsblatts Rottenburg haben bis 1978 insgesamt 900.000 DM zur Errichtung einer Heimstatt geistig behinderter Kinder bereitgestellt ${ }^{28}$.

Werfen wir auch einen Blick auf die Umsatzerlöse der konfessionellen Presse, die der Geschäftsführer der Medien-Dienstleistungsgesellschaft (MDG), Raimund Brehm, für das Jahr 1978 erstellt hat ${ }^{29}$. Er geht dabei von einer rechnerischen Wochenauflage von 6,86 Millionen Exemplaren aus, davon entfallen nach dieser Rechnung 4,26 Millionen Exemplare auf die katholische und 2,6 Millionen Exemplare auf die evangelische Presse. Der Hauptanteil entfällt mit 2,642 Millionen Exemplaren auf die regionalen Kirchenzeitungen (22 Bistumszeitungen, 14 evangelische Landeskirchenzeitungen, wobei die Bistumsblätter eine Auflage von rund einer Million Exemplaren mehr haben):

Die konfessionelle Presse erzielte danach 1978 einen Gesamtumsatz von 260.000 .000 DM. Aufgeschlüsselt ergibt dies für die Kirchenzeitungen 105.000.000 DM, für die übrigen Zeitschriften 155.000.000 DM. Nach Konfessionen berechnet erzielte die Katholische Presse 180.000.000 DM und die evangeolische 80.000.000 DM Umsatz.

Vergleicht man die 120 wöchentlich erscheinenden Publikumszeitschriften (Stand 1975) mit einer Auflage von 31 Millionen Exemplaren mit den 36 Kirchenzeitungen (2,64 Millionen Exemplare, gleich 8,5 Prozent), so meint Brehm, könne man schwerlich von einem Riesen sprechen. Andererseits findet er: „Bei aller Unterschiedlichkeit katholischer und evangelischer Presse ist darin das Wesentliche und Spezifische konfessioneller Presse zu sehen: Der Vertrauenskontakt zwischen $\mathrm{Re}-$ daktion und Leser und die hohe Identifikation mit dem Objekt, die sich in einer starken meinungsbildenden Kraft dieser Presse niederschlägt. So gesehen ist die lebensgestaltende und orientierende Wirkung konfessioneller Presse sicher stärker 
zu gewichten als die Effizienz mancher publikumswirksamer Reichweiten-Riesen, deren Bedeutung als Meinungsbildner vielfach überschätzt wird." ${ }^{30}$

Das hat auch Konsequenzen für das Anzeigengeschäft der konfessionellen Presse, deren wichtigste Blätter sich in der KONPRESS-Anzeigen eG zusammengeschlossen haben. Diese Gesellschaft konnte 1979 gegenüber 1978 ihren Gesamtumsatz um 19,45 Prozent steigern und erzielte damit ihr bislang bestes Jahresergebnis. Bei den Anzeigen klettete der Agentur-Netto-Umsatz von 3.146,919 auf 4.025.411 DM, was 27,9 Prozent entspricht. Im Beilagenbereich konnte eine Steigerung um 9,8 Prozent erzielt werden, was 3.024.438 DM entspricht. Damit überstieg der Gesamtumsatz die 7-Millionen-DM-Grenze.

Trotz der erheblich angestiegenen Anstrengungen und des gewachsenen Bewußtseins für die Notwendigkeit katholischer Presse bleibt für die nächsten Jahre noch viel zu tun. Im Vordergrund und Mittelpunkt muß die Sorge um eine bessere redaktionelle Leistung der Kirchenzeitungen stehen, die engere Leser-Blatt-Bindung, die Steigerung der Auflagen. Dabei handelt es sich um eine Gemeinschaftsaufgabe aller - vom Redakteur zum Verleger, vom Pfarrer zum Bischof, vom katholischen Medienverantwortlichen bis zum einzelnen Leser. Die Sache der katholischen Zeitschrift hat diese Gemeinschaftsanstrengung verdient.

\section{Anmerkungen}

* Teil I erschien unter dem Titel „Die Zukunft kehrt zurück” (CS 12:1979, S. 13 ff.) und Teil II unter dem Titel „Noch schwankt der Boden” (CS 12:1979, S. 244 ff); die Untersuchung wird hier mit Teil III unter dem Titel „Die Anstrengungen lohnen sich” abgeschlossen.

1 De instrumentis informationis. 25 Jahre Katholische Nachrichtenagentur. 3. erweiterte und überarbeitete Auflage. München-Bonn 1977, S. 6/7

2 Ebd., S. 18.

3 Hans-Dieter Wolfinger, „ZV + ZV” 44-45/1978.

4 Dieser Betrag wird allgemein genannt, obwohl sich die offizielle Kirche über den Kaufpreis ausschweigt.

5 De instrumentis informationis, a.a.O. S. 18.

6 Ebd. S. 3.

7 Ebd. S. 4/5.

8 Ebd. S. 6/7.

9 Ebd. S. 10/11.

10 Gesamtplan der evangelischen Publizistik ist Ende 1979 als Buch für 19,80 DM unter dem Titel „Publizistischer Gesamtplan der Evangelischen Kirche in Deutschland” in Gütersloh erschienen.

11 De instrumentis informationis, a.a.O. S. 37.

12 Ebd. S. 28.

13 Die folgenden Angaben beruhen auf einem Telefongespräch mit Dr. Konrad Kraemer Mitte März 1980. 
14 Nach KNA-Angaben.

15 Telefonische Auskunft März 1980.

16 Heinz Wilhelm Brockmann: Die rheinische Meinungsbastion, „Publik-Forum” v. 2. November 1979.

17 AKP-Geschäftsführer Bruno Geute in einem Telefongespräch März 1980.

18 Nach AKP-Amgaben.

19. Rechenschaftsbericht auf der 29. Mitgliederversammlung der AKP am 12. Oktober 1978 in Augsburg.

20 Ebd.

21 Ebd.

22 Horst Schwebel: Glaubwürdig. Fünf Gespräche über heutige Kunst und Religion. München 1979, S. 54.

23 AKP-Mitteilungen II/1979, S. 14.

${ }^{24}$ Rechenschaftsbericht, a.a.O. Zur neuen Werbegesellchaft vgl. AKP-Mitteilungen I/1980, S. $8 \mathrm{f}$.

25 Lt. Telefongespräch vom März 1980.

26 Vgl. AKP-Mitteilungen V/78 und I/79.

27 Als Manuskript gedruckt Februar 1979 unter dem Titel „Nutzung und Beurteilung des Vertriebsweges Schriftenstand durch die katholische Presse”.

28 AKP-Mitteilungen I/79.

29 Raimund Brehm: Die konfessionelle Presse: David oder Goliath im Pressemarkt, „ZV+ ZV", 44-45/1978, S. $1785 \mathrm{ff}$.

30 Ders.: Die konfessionelle Presse, a.a.O.

\section{SUMMARY}

In the past decade the Catholic Church in Germany has made every effort to solve the crisis of the Catholic Press. After the closure of the weekly "Publik", Catholic Media planners directed their attention to the Diocesan weeklies and publications such as "Rheinischer Merkur" (weekly) and "Weltbild" (bi-monthly), and to the strengthening of the Catholic News Agency (KNA) and improving training. This was necessary on account of the decrease in circulation and the gap between the media and the Church. One of the most important developments in Catholic Communications work has been the establishment of a Media Service Company (Medien-Dienstleistungsgesellschaft - MDG) in Munich in 1975. This was part of the communications emergency planning at the Synod of the German Dioceses in 1972. The association of the Catholic Press - AKP - also helped towards a revival. New and improved cooperation among the diocesan weeklies - important instruments of dialogue within the Church was developed. The author, in describing these developments, does not confine himself to publications, but also refers to press officers of the diocese and to publications from religious communities and missionary societies which have an important role in the press today. $\mathrm{He}$ feels that the crisis of the Catholic Press has been overcome, but that a strenuous effort is needed to implant this same press firmly into the German secular communications media. 


\section{RÉSUMÉ}

Au cours de la derniére décennie, l'Eglise catholique fit des efforts considérables pour la presse catholique en crise. Après la suppression de l'hebdommadaire «Publik», la politique de mass media de l'Eglise consécra au renforcement des journaux épiscopaux, à la préservation du (Rheinischer Merkur» et du "Weltbild», de même que d'autres journaux épiscopaux, et objets d'édition, au développement de l'agence de presse catholique (KNA) de même que l'encouragement de la relève journalistique. Ceci fut nécessaire parce que l'attachement à l'Eglise et avec lui le nombre d'exemplaires baissaient. Parmi les principales nouveautés sur le marché des mass media catholique on compte aussi la société de services des mass media (MDG) - fondée en 1975 - à Munich comme partie du «programme publicistique express» et qui fut décidée en 1972 lors du synode commun des évêchés allemands. La communauté de travail de la press catholique (AKP) fait aussi partie des aides à l'intérieur des journaux épiscopaux qui, depuis des années, sont en train de changer fondamentalement. Ils prennent une part importante au dialogue à l'intérieur de l'Eglise. L'auteur expose les développements et ambitions avec le conflit d'opinions. En cela, il ne se limite pas seulement aux journaux épiscopaux, mais il aborde aussi les bureaux de presse des évêchés, de même que la presse des ordres et des missions qui est également une partie intégrante importante de la presse catholique actuelle. Il est persuadé que la crise proprement dite de la presse catholique est actuellement surmontée. De gros efforts sont assurément encore nécessaires pour assurer à la presse catholique la place qui lui revient à l'intérieur du paysage des mass media allemands.

\section{RESUMEN}

En el último decenio la Iglesia católica alemana hizo notables esfuerzos en pro de la prensa católica en crisis. Después de la supresión del semanario «Publik», la política eclesial para medios de comunicación trato de robustecer la prensa diocesana, de asegurar al periodico «Rheinischer Merkur» y a la revista «Weltbild», así como a otras revistas y editoras, ampliando al mismo tiempo la Agencia Noticiosa Católica (KNA) y promocionando las vocaciones periodísticas. Todo ello fué necesario porque desminuyó el vínculo eclesial y con ello el número de suscriptores. Entre las más importantes novedades en el mercado de los medios de comunicación católicos se encuentra la fundación en 1975 de la MDG, una empresa de servicios para la comunicación social creada en Munich en el marco del «Programa Publicistico de Urgencia», aprobado en 1972 por el Sinodo Conjunto de las Diócesis Alemanas. También el Equipo de trabajo Prensa Católica (AKP) es uno de los puntales del publicismo católico en Alemania. Como consecuencia de estos esfuerzos surgió una estrecha colaboración entre los periódicos diocesanos, que se encuentran en evolución desde las años. Esta prensa participa de forma importante en el diálogo interno eclesial. El autor presenta el desarrollo y aspiraciones, junto con el antagonismo de las opiniones. No se limita a la prensa diocesana: se ocupa también de las oficinas de prensa episcopales, así como de la prensa misionera y de las órdenes religiosas, que es parte importante de la prensa católica actual. Está convencido de que fué superada ya la crisis de la prensa católica. Sin embargo son necesarios todavía grandes esfuerzos para asegurar a la prensa católica el puesto que le corresponde en el panorama publicístico alemán. 